\title{
Efeito da goma de mascar contendo sacarose e do dentifrício fluoretado na remineralização in situ de lesões de cárie artificiais
}

\section{Effect of sucrose-containing gum and fluoridated dentifrice on in situ remineralization of caries lesions}

\author{
Renata Rodrigues de FREITAS* \\ Jonas Alves de OLIVEIRA** \\ Eulázio Mikio TAGA*** \\ Marília Afonso Rabelo BUZALAF****
}

\begin{abstract}
FREITAS, R. R. de; OLIVEIRA, J. A. de; TAGA, E. M.; BUZALAF, M. A. R. Efeito da goma de mascar contendo sacarose e do dentifrício fluoretado na remineralização in situ de lesões de cárie artificiais. Pesqui Odontol Bras, v. 15, n. 2, p. 98-103, abr./jun. 2001.
\end{abstract}

\begin{abstract}
Este trabalho objetivou avaliar a remineralização de lesões iniciais de cárie in situ em esmalte bovino. Produziram-se as lesões artificialmente com o uso de solução desmineralizante, fixando-as na região correspondente à superfície lingual de primeiros molares inferiores, através de aparelhos removiveis, em seis voluntários com idades entre 18 e 22 anos, seguindo-se três períodos experimentais de uma semana. No primeiro período (grupo controle), realizou-se a escovação com dentifrício sem flúor, 4 vezes ao dia, após as refeições; no segundo (grupo I), procedeu-se à escovação com dentifrício contendo 1.500 ppm de flúor na forma MFP, 4 vezes ao dia, após as refeições; e no terceiro (grupo II), escovação com dentifrício sem flúor e utilização de goma de mascar contendo $60 \%$ de sacarose, por 20 minutos, 4 vezes ao dia, após as refeições. Antes e depois de cada tratamento, realizaram-se ensaios de dureza Vickers (200 g), obtendo-se o percentual de remineralização de cada lesão $(\alpha)$. O grupo controle sofreu uma desmineralização de $2,78 \%$, e os grupos I e II, remineralização de 3,36\% e 5,21\%, respectivamente. A análise estatística através dos testes de Kruskal-Wallis e de Miller revelou uma diferença significante $(\mathrm{p}<0,05)$ entre o grupo controle e os experimentais (I e II). Entretanto, apesar de o grupo II sofrer maior remineralização em relação ao grupo I, esta diferença não foi significante. Estes resultados sugerem que o uso da goma de mascar com sacarose e do dentifrício fluoretado pode ter efeito considerável na remineralização de lesões iniciais de cárie, tornando-se uma alternativa viável em sua prevenção.
\end{abstract}

UNITERMOS: Remineralização dentária; Dureza; Goma de mascar; Dentifrícios; Flúor.

\section{INTRODUÇÃO}

Atualmente, vêm sendo empregadas técnicas laboratoriais e clínicas para o estudo da mineralização do esmalte ou dentina, em modelos intrabucais, acompanhando a perda e/ou ganho do mineral no desenvolvimento do processo carioso por pequenos periodos ${ }^{15}$.

Desde que observações clínicas comprovaram que lesões de mancha branca são reversiveis, a remineralização passou a ser um importante mecanismo na prevenção e redução clínica das cáries em esmalte ${ }^{2,3,4,6,7,10,11,13,14,15,17,19,20,21,22,23}$. Assim sendo, fatores que aumentem o fluxo salivar e estimulem a mineralização têm efeito cárie-protetor, como os anti-sépticos bucais, dentifrícios e géis fluoreta- dos, alternativas consideráveis no controle da cárie por auxiliarem o processo natural de remineralização ${ }^{10}$.

A goma de mascar, mesmo contendo sacarose, aumenta a capacidade tampão da saliva devido ao aumento do fluxo salivar, diminuindo assim o acúmulo de placa ${ }^{1,6,7,14,15,16,17,18,19,23}$, mantendo os níveis do $\mathrm{pH}$ e, conseqüentemente, neutralizando os efeitos deletérios dos ácidos produzidos pela presença de carboidratos fermentáveis na alimentação ${ }^{10,18}$.

O presente estudo tem como objetivo analisar a influência da goma de mascar com sacarose, do dentifrício fluoretado e da própria saliva na remineralização de lesões artificiais de cárie em esmalte bovino in situ.

\footnotetext{
${ }^{*}$ Cirurgiã-Dentista.
}

**Graduando em Odontologia; ***Professor Titular do Departamento de Ciências Biológicas; ****Professora Assistente Doutora do Departamento de Ciências Biológicas - Faculdade de Odontologia de Bauru da USP. 
FREITAS, R. R. de; OLIVEIRA, J. A. de; TAGA, E. M.; BUZALAF, M. A. R. Efeito da goma de mascar contendo sacarose e do dentifrício fluoretado na remineralização in situ de lesões de cárie artificiais. Pesqui Odontol Bras, v. 15, n. 2, p. 98-103, abr./jun. 2001.

\section{MATERIAL E MÉTODOS Preparo dos espécimes}

Incisivos bovinos foram seccionados com discos diamantados, sendo obtidos blocos de esmalte de 4 x $4 \mathrm{~mm}$.

Os espécimes obtidos do mesmo dente foram selados com esmalte de unha, exceto na superficie que seria exposta ao meio bucal e inclusos num mesmo corpo-de-prova de resina epóxica. Foi dado polimento superficial gradativo com lixas de carbeto de tungstênio de números 400, 600 e 1.200 acopladas a politriz DP $9^{A}$ (Panambra Ind. e Tecn. S. A.).

A desmineralização dos espécimes foi realizada com os dentes ainda inclusos. Para cada fragmento, utilizaram-se $20 \mathrm{ml}$ da solução desmineralizante proposta por WANG et $a ._{.}{ }^{21}$, que consistia de tampão ácido lático/hidróxido de sódio $0,1 \mathrm{M}$, contendo carboximetilcelulose sódica a $1 \%, \mathrm{Ca}^{+2}$ $3 \mathrm{mM}, \mathrm{PO}_{4}^{-3} 1,8 \mathrm{mM}, \mathrm{F}^{-} 0,263 \mu \mathrm{M}, \mathrm{pH} 4,0$. Os fragmentos foram imersos nesta solução e conservados em estufa em temperatura constante de $37^{\circ} \mathrm{C}$ por 39 horas.

Os espécimes foram removidos da solução desmineralizante, lavados intensamente em água deionizada e esterilizados com gás Oxyfume-12 (White Martins), à base de óxido de etileno. A esterilização foi realizada a $39^{\circ} \mathrm{C}$ por $24 / 48$ horas dependendo do número de espécimes contidos no corpo-de-prova.

\section{Procedimentos intrabucais}

Foram selecionados seis adultos jovens saudáveis, com idades entre 18 e 22 anos, os quais foram devidamente esclarecidos sobre os objetivos e a metodologia a ser empregada no estudo, tendo assinado um termo de consentimento livre e esclarecido concordando em participar do mesmo, onde ficava claro que a qualquer momento poderiam desistir de participar do experimento e que os dados obtidos seriam confidenciais. Os voluntários foram submetidos a exame clínico e radiográfico nos períodos pré- e pós-experimento. Os voluntários receberam dispositivos intra-orais de resina acrílica (Figura 1) contendo 2 espécimes.

Cada voluntário usou o aparelho continuamente, exceto durante as refeições, por 3 períodos experimentais de 7 dias, separados por um intervalo de 7 dias ("wash-out") entre eles:

- grupo controle: os pacientes foram orientados a não utilizar goma de mascar e a realizar a esco-

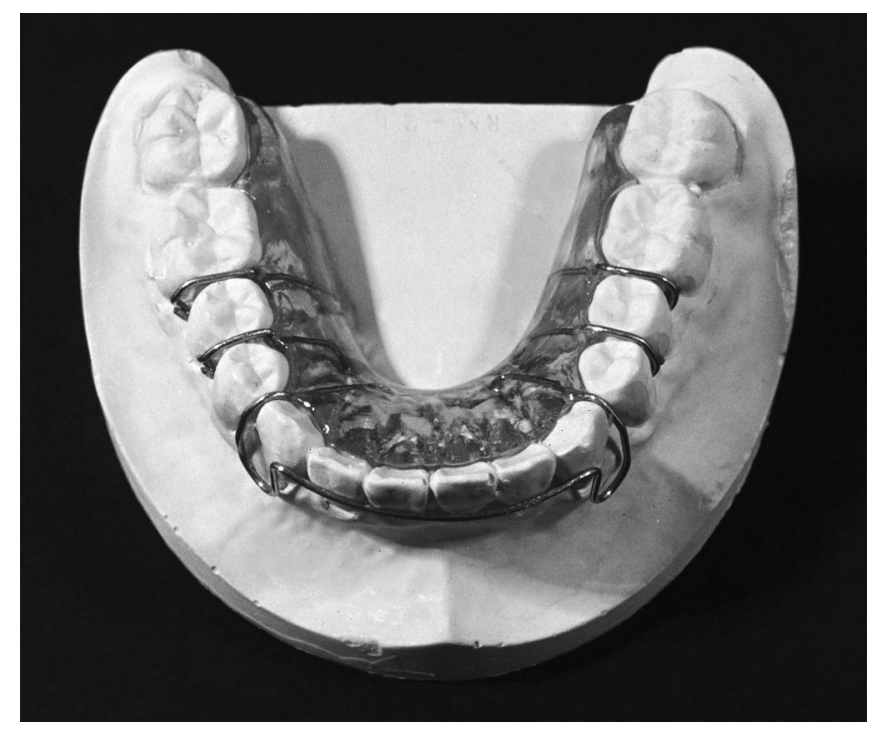

FIGURA 1 - Modelo do aparelho usado.

vação com dentifrício não fluoretado formulado pela Kolynos do Brasil S. A. (KB-1080-1-29);

- grupo I: os voluntários foram orientados a não usar goma de mascar e a realizar a escovação dental com dentifrício contendo 1.500 ppm de F na forma de MFP (Sorriso, Kolynos do Brasil S. A.), 4 vezes ao dia, após as refeições;

- grupo II: os voluntários foram orientados a mascar a goma (Spin, Sukest) contendo 60\% de sacarose, 4 vezes ao dia (após as refeições) por 20 minutos. A escovação foi realizada, após o uso da goma, com dentifrício não fluoretado (KB-1080-1-29, Kolynos do Brasil S. A.).

Este trabalho teve a aprovação do Comitê de Ética em Pesquisa do Hospital de Reabilitação de Anomalias Craniofaciais da USP - Bauru, São Paulo.

\section{Análise da microdureza}

As análises de microdureza foram realizadas com o M-Testor 337, ao qual foi acoplada uma ponta piramidal (dureza Vickers), usando-se uma carga de 200 g para fazer as indentações. Na parte central de cada espécime, foram feitas três indentações, a partir das quais calculou-se uma média, que foi convertida para os valores de dureza correspondentes. Em cada espécime, foram feitas três medições, a saber:

- DH: primeira análise, correspondente ao esmalte hígido após o polimento da superfície;

- DD: segunda análise, correspondente ao esmalte desmineralizado;

- DT: terceira análise, realizada após o período 
FREITAS, R. R. de; OLIVEIRA, J. A. de; TAGA, E. M.; BUZALAF, M. A. R. Efeito da goma de mascar contendo sacarose e do dentifrício fluoretado na remineralização in situ de lesões de cárie artificiais. Pesqui Odontol Bras, v. 15, n. 2, p. 98-103, abr./jun. 2001.

TABELA 1 - Valores médio, mínimo e máximo e desvio-padrão de DH (primeira análise), DD (segunda análise), DT (terceira análise) e $\alpha$ (porcentagem de remineralização).

\begin{tabular}{c|c|c|r|r|r|r}
\hline \hline Grupos & Variável & $\mathrm{n}$ & Média & Mínimo & Máximo & Desvio-padrão \\
\hline \multirow{4}{*}{ Controle } & $\mathrm{DH}$ & 10 & 583,300 & 476,000 & 683,000 & 55,33745 \\
\cline { 2 - 7 } & $\mathrm{DD}$ & 10 & 85,030 & 74,400 & 95,300 & 7,63094 \\
\cline { 2 - 7 } & $\mathrm{DT}$ & 10 & 71,180 & 50,700 & 111,000 & 18,55669 \\
\cline { 2 - 7 } & $\alpha$ & 10 & $-2,798$ & $-7,580$ & 3,190 & 3,45125 \\
\hline \multirow{4}{*}{ Grupo I } & $\mathrm{DH}$ & 10 & 592,800 & 458,000 & 653,000 & 55,66527 \\
\cline { 2 - 7 } & $\mathrm{DD}$ & 10 & 66,950 & 60,200 & 71,900 & 3,71611 \\
\cline { 2 - 7 } & $\mathrm{DT}$ & 10 & 86,910 & 75,000 & 97,100 & 8,51697 \\
\hline \multirow{5}{*}{ Grupo II } & $\alpha$ & 10 & 3,824 & 1,080 & 6,430 & 2,05166 \\
\cline { 2 - 7 } & $\mathrm{DH}$ & 10 & 553,500 & 451,000 & 631,000 & 54,61634 \\
\cline { 2 - 7 } & $\mathrm{DD}$ & 10 & 52,520 & 45,800 & 57,700 & 4,43942 \\
\cline { 2 - 7 } & $\mathrm{DT}$ & 10 & 75,750 & 63,900 & 98,200 & 11,97852 \\
\hline \hline
\end{tabular}

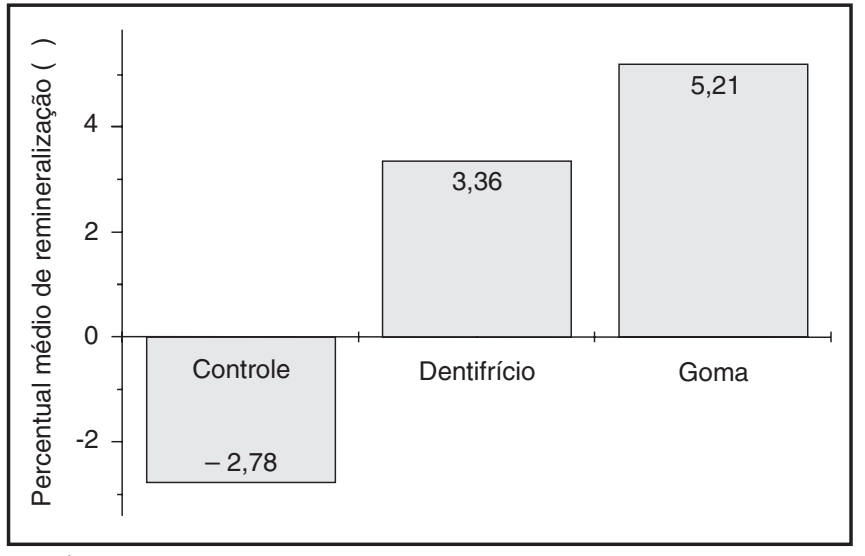

GRÁFICO 1 - Percentual médio de remineralização $(\alpha)$ dos grupos controle, I (dentifrício) e II (goma).

experimental, subdividida em DC, DI e DII, correspondendo aos grupos controle, I e II, respectivamente.

A porcentagem de remineralização $(\alpha)$ foi calculada a partir da seguinte fórmula:

$$
\alpha=\frac{D T-D D}{D H-D D}
$$

\section{Análise estatística}

A análise estatística dos dados foi realizada sobre a variável $\alpha$, através do teste de Kruskal-Wallis e o teste de Miller foi feito para comparações individuais entre os grupos, em nivel de significância de $5 \%$.
TABELA 2 - Teste de Kruskal-Wallis em função de $\alpha$ (porcentagem de remineração).

\begin{tabular}{c|c|c}
\hline \hline Grupos & $\mathrm{n}$ & Posto médio \\
\hline Controle & 10 & 64,0 \\
\hline I & 10 & 194,0 \\
\hline II & 10 & 207,0 \\
\hline \hline
\end{tabular}

\section{RESULTADOS}

A Tabela 1 mostra os valores máximos e mínimos de dureza Vickers através das variáveis $\mathrm{DH}$, DD e DT, e de $\alpha$, além da média e do desvio-padrão dos espécimes dos grupos controle, I (dentifrício fluoretado) e II (goma de mascar), respectivamente. Conforme podemos observar, no grupo controle houve desmineralização, com valores de $\alpha$ entre $-7,58 \%$ e $3,19 \%$. Nos grupos I e II houve remineralização, com valores de $\alpha$ entre $1,08 \%$ e $6,43 \%$ para o grupo I e entre $1,42 \%$ e $10,45 \%$ para o grupo II.

O Gráfico 1 representa o $\alpha$ médio encontrado para os grupos controle, I e II. Nota-se que o grupo controle sofreu uma desmineralização de 2,78\% durante o período experimental, e os grupos I e II apresentaram remineralização de 3,36 e 5,21\%, respectivamente.

O teste de Kruskal-Wallis revelou que existe diferença significante entre os grupos (Tabela 2), e a partir do teste de Miller para comparações individuais, encontrou-se que, no nível de significância 
FREITAS, R. R. de; OLIVEIRA, J. A. de; TAGA, E. M.; BUZALAF, M. A. R. Efeito da goma de mascar contendo sacarose e do dentifrício fluoretado na remineralização in situ de lesões de cárie artificiais. Pesqui Odontol Bras, v. 15, n. 2, p. 98-103, abr./jun. 2001.

TABELA 3 - Teste de Miller para comparações individuais.

\begin{tabular}{l|c}
\hline \hline \multicolumn{1}{c|}{ Comparação } & Diferença de posto médio \\
\hline Controle versus grupo I & $13,00^{*}$ \\
\hline Controle versus grupo II & $14,30^{*}$ \\
\hline Grupo I versus grupo II & 1,30 \\
\hline \hline
\end{tabular}

*Significante $(\mathrm{p}<0,05)$. Valor crítico para comparação: 9,23 .

de $5 \%$, houve diferença significante entre os grupos controle e I, e entre o controle e II. O grupo II revelou maior remineralização que o grupo I, mas sem diferença significante (Tabela 3 ).

\section{DISCUSSÃO}

No presente estudo, foi analisado o potencial remineralizante de um dentifrício fluoretado contendo $1.500 \mathrm{ppm}$ de flúor e de uma goma de mascar contendo $60 \%$ de sacarose, através da medição da microdureza superficial do esmalte, que é bastante sensivel na detecção do amolecimento superficial do esmalte e pode ser aplicado no mesmo espécime antes e depois de um determinado tratamento, o que permite quantificar o ganho ou a perda mineral superficial indiretamente ${ }^{15}$.

GELHARDS; ARENDS ${ }^{11}$ (1984) relataram que a taxa de remineralização é alta nas primeiras duas semanas, diminuindo gradativamente em períodos mais longos. Por este motivo, optou-se pelo período experimental de 7 dias, apesar de vários autores empregarem periodos experimentais mais lon$\operatorname{gos}^{1,5,6,11,21}$. Este período foi suficiente para permitir a detecção de remineralização superficial das lesões nos grupos experimentais, mas a deposição de mineral na profundidade da lesão só poderia ser avaliada por outros métodos quantitativos, como a microrradiografia ${ }^{16}$. Entretanto, vários autores concordam que a deposição de mineral é gradativa e se dá mais rapidamente na superfície que em partes mais profundas da lesão $0^{4,11,13,17,18}$. Acredita-se que os depósitos em profundidade são retardados pela presença da película adquirida e pela deposição mais externa do mineral, restringindo a difusão iônica em profundidade ${ }^{4,7}$. MANNING; $\operatorname{EDGAR}^{15}$ (1991) associaram este fato ao pequeno período experimental. Seria interessante fazer estudos futuros empregando-se períodos mais longos, a fim de se tentar obter percentuais de remineralização maiores.

O uso de gomas de mascar aumenta o fluxo salivar devido ao estímulo mastigatório e gustativo.
Essa elevação do fluxo salivar, na ausência de uma produção significante de ácidos, como ocorre nas gomas adoçadas com sorbitol e xilitol, aumenta o $\mathrm{pH}$ da saliva e da placa, a quantidade e a concentração de cálcio secretado e, secundariamente ao aumento do $\mathrm{pH}$, a concentração do fosfato ${ }^{7,14}$. CREANOR et al. ${ }^{6}$ (1992) relataram que o estímulo do fluxo salivar pela goma atinge um pico de cerca de 10 vezes o fluxo não estimulado no primeiro minuto, ocorrendo então um rápido decréscimo até um platô correspondendo a 3 vezes o fluxo não estimulado depois de 20 minutos, sendo que o padrão de fluxo salivar é o mesmo, empregando-se gomas de diferentes sabores, adoçadas com sacarose ou com seus substitutos. No caso de gomas contendo sacarose, a concentração do açúcar na saliva atinge um pico nos primeiros 1 a 2 minutos e então cai rapidamente. Acredita-se que essa breve exposição adicional a ácidos, como conseqüência da elevação inicial dos niveis de sacarose contribui para a limpeza dos poros superficiais da lesão, permitindo o acesso dos minerais às porções mais profundas, o que levaria a um padrão de deposição mineral mais desejável.

EDGAR et $a .^{7}$ (1975), usando goma de mascar por 5 minutos, observaram que aquelas contendo sorbitol tinham um efeito muito pequeno no $\mathrm{pH}$ da placa, enquanto as gomas contendo sacarose o diminuíam. Isso pode ser atribuído ao pequeno período de utilização dessas gomas, pois MANNING; $\operatorname{EDGAR}^{15}$ (1991) relataram que a utilização de gomas de mascar contendo ou não sacarose depois de um desafio acidogênico diminuía o tempo para o $\mathrm{pH}$ voltar aos níveis do repouso.

CREANOR et al. ${ }^{6}$ (1992) observaram que o uso de gomas contendo sacarose e sorbitol tinha efeito semelhante, na superficie do esmalte, mas no corpo da lesão, as com sacarose promoviam maior remineralização. Em nosso estudo, empregamos goma de mascar contendo sacarose por 20 minutos após as refeições e observamos uma remineralização significantemente maior em relação ao controle já em uma semana. Seria interessante fazer um estudo comparando-se gomas contendo sacarose com aquelas contendo seus substitutos, a fim de se observar se haveria diferenças no percentual de remineralização, conforme relatado por alguns autores.

LEACH et al. ${ }^{14}$ (1989) empregaram gomas contendo sorbitol por 20 minutos após as refeições e 2 lanches intermediários e observaram que o potencial de remineralização pela saliva dobrou em 3 semanas, mas nesse estudo, os pacientes usavam dentifrício fluoretado 2 vezes por dia, pondo em 
FREITAS, R. R. de; OLIVEIRA, J. A. de; TAGA, E. M.; BUZALAF, M. A. R. Efeito da goma de mascar contendo sacarose e do dentifrício fluoretado na remineralização in situ de lesões de cárie artificiais. Pesqui Odontol Bras, v. 15, n. 2, p. 98-103, abr./jun. 2001.

dúvida o potencial remineralizante da goma. Por este motivo, em nosso estudo empregamos 2 grupos experimentais, sendo que um deles utilizou goma contendo sacarose e dentifrício não fluoretado, e o outro, dentifrício fluoretado sem a goma, a fim de se analisar o potencial remineralizante isoladamente. Nos dois casos, houve uma remineralização estatisticamente significante em relação ao controle, sem entretanto haver diferença entre os grupos experimentais no período de uma semana.

CREANOR et al. ${ }^{5}$ (1986) acompanharam a remineralização por dentifrício contendo ou não flúor, e coincidindo com os resultados por nós obtidos, após uma semana, houve desmineralização no grupo que utilizou dentifrício sem flúor.

\section{CONCLUSÕES}

Os resultados obtidos demonstraram que o uso de dentifrício contendo $1.500 \mathrm{ppm}$ de flúor e de goma de mascar contendo sacarose aumenta significativamente a porcentagem de remineralização de lesões cariosas incipientes produzidas artificialmente. Seria interessante a realização de estudos complementares, prolongando-se o período experimental, bem como comparando-se os efeitos de gomas de mascar contendo ou não sacarose para que alguns aspectos possam ser melhor esclarecidos.

\section{AGRADECIMENTOS}

Ao PIBIC-CNPq, pelo auxílio finaceiro. À Colgate do Brasil, pela cessão dos dentifrícios. À Sukest, pelo fornecimento de gomas de mascar. Aos Professores do Departamento de Dentística/Materiais Dentários da FOB-USP, pelo auxílio na realização dos ensaios de microdureza. Ao Professor Dr. Eymar Sampaio Lopes, pela realização da análise estatística.

\footnotetext{
FREITAS, R. R. de; OLIVEIRA, J. A. de; TAGA, E. M.; BUZALAF, M. A. R. Effect of sucrose-containing gum and fluoridated dentifrice on in situ remineralization of caries lesions. Pesqui Odontol Bras, v. 15, n. 2, p. 98-103, abr./jun. 2001.
}

\begin{abstract}
The aim of this study was to evaluate the remineralization of incipient carious lesions in bovine enamel in situ. Artificial carious lesions were produced and fixed in removable lower appliances in the region of the lingual surfaces of first molars, in six volunteers with ages between 18 and 22 years, who were subjected to 3 distinct experimental periods of 1 week each. In the first period (control group), patients brushed their teeth with a non-fluoridated dentifrice 4 times a day (after meals), and, in the second period (group I), patients used a dentifrice containing 1,500 ppm of fluorine (in the form of MFP). In the third period (group II) volunteers brushed their teeth with non-fluoridated dentifrice and used chewing gum containing $60 \%$ of sucrose during 20 minutes, 4 times a day (after meals). Before and after each treatment, the specimens underwent Vicker's hardness test (200 g of load), and the remineralization percentage $(\alpha)$ was calculated. The control group showed $2.78 \%$ of demineralization, and groups I and II showed 3.36 and $5.21 \%$ of remineralization, respectively. Statistical analysis (with Kruskal-Wallis and Miller's tests) showed significant difference $(\mathrm{p}<0.05)$ between the control and experimental groups (I and II). Group II showed greater $\alpha$ than group I, but this difference was not significant. These results suggest that the use of sucrose-containing chewing gum and fluoridated dentifrice has a considerable effect on the remineralization of incipient carious lesions and may be a valuable alternative for their prevention.
\end{abstract}

UNITERMS: Tooth remineralization; Hardness; Chewing gum; Dentifrices; Fluorine.

\section{REFERÊNCIAS BIBLIOGRÁFICAS}

1. AGUIRRE-ZERO, O.; ZERO, D. T.; PROSKIN, H. M. Effect of chewing xylitol chewing gum on salivary flow rate and the acidogenic potential of dental plaque. Caries Res, v. 27, n. 1, p. 55-59, 1993.

2. ARENDS, J.; SCHUTOF, J.; JONGEBLOED, W. G. Lesions depth and microhardness indentations on artificial white spot lesions. Caries Res, v. 14, p. 190-195, 1980.

3. BOWEN, W. H.; PEARSON, S. K. The effects of sucrose, xylitol and sorbitol on remineralization of caries lesions in rats. J Dent Res, v. 71, n. 5, p. 1166-1168, 1992.

4. BRUDEVOLD, F.; ATTARZADEH, F.; TEHRANI, J. V. H. et al. Development of a new intraoral demineralization test. Caries Res, v. 18, p. 421-429, 1984.
5. CREANOR, S. L.; STRANG, R.; TELFER, S. et al. In situ appliance for the investigation of enamel de- and remineralization. Caries Res, v. 20, p. 385-391, 1986.

6. CREANOR, S. L.; STRANG, R.; GILMOUR, W. H. et al. The effect of two chewing-gum use on in situ enamel lesion remineralization. J Dent Res, v. 71, n. 12, p. 1895-1900, 1992.

7. EDGAR, W. M.; BIBBY, B. G.; MANDORFF, S. et al. Acid production in plaques after eating snacks modifying factors in food. J Am Dent Assoc, v. 90, p. 418-425, 1975.

8. EDGAR, W. M. Sugar substitutes, chewing-gum and dental caries - a review. Br Dent J, v. 184, n. 1, p. 29-32, 1998.

9. EXTERCATE, R. A. M.; DAMEN, J. J. M.; TEN CATE, J. M. A single-section model for de- and remineralization studies. I - defects of different $\mathrm{Ca} / \mathrm{P}$ ratios in remineraliza- 
FREITAS, R. R. de; OLIVEIRA, J. A. de; TAGA, E. M.; BUZALAF, M. A. R. Efeito da goma de mascar contendo sacarose e do dentifrício fluoretado na remineralização in situ de lesões de cárie artificiais. Pesqui Odontol Bras, v. 15, n. 2, p. 98-103, abr./jun. 2001.

tion solutions. J Dent Res, v. 72, n. 12, p. 1599-1603, 1993.

10. FEATHERSTONE, J. D. B.; CUTRESS, T. W.; RODGERS, B. E. et al. Remineralization of artificial caries-like lesions in vivo by a self-administered mouthrinse or paste. Caries Res, v. 16, p. 235-242, 1982.

11. GELHARDS, T. B. F. M.; ARENDS, J. In vivo remineralization of artificial subsurface lesions in human enamel - I. J Biol Buccale, v. 12, p. 49-57, 1984.

12. GLASS, R. L. A two-year clinical trial of sorbitol chewing gum. Caries Res, v. 17, p. 365-368, 1983.

13. HOLMEN, L.; THYLSTRUP, A.; ARTUN, J. Clinical and histological features observed during arrestment of active enamel carious lesions in vivo (with 1 color plate). Caries Res, v. 21, p. 546-554, 1987.

14. LEACH, S. A.; LEE, G. T. R.; EDGAR, W. M. et al. Remineralization of artificial caries-like lesions in human enamel in situ by chewing sorbitol gum. J Dent Res, v. 68, n. 6, p. 1064-1068, 1989.

15. MANNING, R. H.; EDGAR, W. M. Effects of chewing gum on plaque $\mathrm{pH}$ profiles after sucrose-containing snack and rinse. Caries Res, v. 25, p. 234, 1991.

16. MANNING, R. H.; EDGAR, W. M. Intra-oral models for studying de- and remineralization in man: methodology and measurement. J Dent Res, v. 71, p. 895-900, April 1992. [Special issue]

17. MANNING, R. H.; EDGAR, W. M.; AGAlAMANYI, E. A.
Effects of chewing gums sweetened with sorbitol or a sorbitol/xylitol mixture on the remineralization of human enamel lesions in situ. Caries Res, v. 26, p. 104-109, 1992.

18. PARK, K. K.; SCHEMEHORN, B. R.; STOOKEY, G. K. Effect of time and duration of sorbitol gum chewing on plaque acidogenicity. Pediatr Dent, v. 15, n. 3, p. 197-201, 1993.

19. STEINBERG, L. M.; ODUSOLA, F.; MANDEL, I. D. Remineralizing potential, antiplaque and antigingivitis effects of xylitol and sorbitol sweetened chewing gum. Clin Prev Dent, v. 14, n. 5, p. 31-34, 1992.

20. VAN HERPEN, B. P. J. M.; ARENDS, J. Mineral distributions in enamel after in vivo de- and remineralization. $\mathbf{J}$ Biol Buccale, v. 15, p. 199-204, 1987.

21. WANG, C. W.; CORPRON, R. E.; LAMB, W. J. et al. In situ remineralization of enamel lesions using continuous versus intermittent fluoride application. Caries Res, v. 27, p. 455-460, 1993.

22. WEFEL, J. S.; MAHARRI, G. J.; JENSEN, M. E. et al. Development of intra-oral single-section remineralization model. J Dent Res, v. 66, n. 9, p. 145-1489, 1987.

23. WENNERHOLM, K.; ARENDS, J.; BIRKHED, D. et al. Effect of xylitol and sorbitol in chewing gums on mutans streptococci, plaque $\mathrm{pH}$ and mineral loss of enamel. Caries Res, v. 28, p. 48-54, 1994.

Recebido para publicação em 10/04/00

Enviado para reformulação em 27/09/00 Aceito para publicação em 21/02/01 